\title{
Human pancreatic $\beta$-cell glucokinase: subcellular localization and glucose repression signalling function in the yeast cell
}

\author{
Alberto RIERA ${ }^{1}$, Deifilia AHUATZI ${ }^{\S}$, Pilar HERRERO ${ }^{1}$, Maria Adelaida \\ GARCIA-GIMENO ${ }^{2,3}$, Pascual SANZ ${ }^{2,3}$ and Fernando MORENO ${ }^{1, *}$
}

${ }^{1}$ Departamento de Bioquímica y Biología Molecular. Universidad de Oviedo, 33006-Oviedo. Spain.

${ }^{2}$ Instituto de Biomedicina de Valencia, Consejo Superior de Investigaciones Científicas (CSIC), Jaime Roig 11, 46010 Valencia, Spain.

${ }^{3}$ CIBER de Enfermedades Raras (CIBERER), Valencia, Spain.

Running title: Glucose repression signalling by pancreatic glucokinase

$\S$ Present address: Departamento de Ingeniería Bioquímica, Escuela Nacional de Ciencias Biológicas, Instituto Politécnico Nacional, 11340-México D.F. México.

* Corresponding author address: Fernando Moreno. Departamento de Bioquímica y Biología Molecular. Campus de El Cristo (Edificio Santiago Gascón). 33006-Oviedo. Spain

Phone: 34-985103567. Fax: 34-985103157. e-mail: fmoreno@uniovi.es 


\section{SUMMARY}

Human pancreatic $\beta$-cell glucokinase $\left(\mathrm{GK}_{\beta}\right)$ is the main glucose phosphorylating enzyme in pancreatic $\beta$-cells. It shares several structural, catalytic and regulatory properties with hexokinase 2 (Hxk2) from Saccharomyces cerevisiae. In fact, it has been previously described that expression of $\mathrm{GK}_{\beta}$ in yeast could replace Hxk2 in the glucose signalling pathway of $S$. cerevisiae. In this study we report that $\mathrm{GK}_{\beta}$ exerts its regulatory role by association with the yeast transcriptional repressor Mig1; the presence of Mig1 allows $\mathrm{GK}_{\beta}$ to bind to the $S U C 2$ promoter, helping in this way in the maintenance of the repression of the $S U C 2$ gene under high-glucose conditions. Since a similar mechanism has been described for the yeast Hxk2, our findings suggest that the function of the regulatory domain present in these two proteins has been conserved throughout evolution. In addition, we report that $\mathrm{GK}_{\beta}$ is enriched in the yeast nucleus of high-glucose growing cells, whereas it shows a mitochondrial localization upon removal of the sugar. However, $\mathrm{GK}_{\beta}$ does not exit the nucleus in the absence of Mig1, suggesting that Mig1 regulates the nuclear exit of $\mathrm{GK}_{\beta}$ under low-glucose conditions. We also report that binding of $\mathrm{GK}_{\beta}$ to Mig1 allows the latter protein to be located at the mitochondrial network under low-glucose conditions.

Keywords: Pancreatic $\beta$-cell glucokinase; Hxk2; Saccharomyces cerevisiae; glucose repression; Mig1

Abbreviations: ChIP, chromatin immunoprecipitation; GAD, Gal4 activating domain; GBD, Gal4 DNA binding domain; GFP, green fluorescent protein; RFP, red fluorescent protein; $\mathrm{GK}_{\beta}, \beta$-cell glucokinase; $\mathrm{GK}_{\mathrm{L}}$, liver glucokinase; GKRP, glucokinase regulatory protein; $\mathrm{SD}$, synthetic complete medium containing glucose as carbon source; 


\section{INTRODUCTION}

Glucokinase (GK), a member of the hexokinase family (hexokinase IV) [1], plays an essential role in glucose phosphorylation in the liver and endocrine $\beta$-cells of the pancreas [2]. GK also acts as a glucose sensor, integrating blood glucose levels and insulin release in pancreatic $\beta$-cells [3] and regulating glucose metabolism in the liver [2]. Hepatic GK shows dynamic nuclear-cytoplasm localization depending on the amount of glucose in blood. The nuclear localization of hepatic GK is regulated by its binding to a glucokinase regulatory protein (GKRP) under low-glucose conditions [4, 5]. In addition, it has been recently described that hepatic GK could also be associated with liver mitochondria [6, 7]. The GK subcellular distribution in the pancreatic $\beta$-cell is also regulated by glucose: low levels of glucose causes an association of GK with mitochondria [8], whereas high glucose levels prevents GK translocation to this organelle [8]. In $\beta$-cells, GK has also been localized on the secretory granules and, although it has been suggested that glucose causes dissociation of glucokinase from this location [9], later studies suggest that glucokinase is an integral component of the granule and does not translocate during glucose stimulation [10].

Pancreatic $\mathrm{GK}_{\beta}$ and the yeast hexokinase 2 (Hxk2) proteins have several structural and functional similarities. For example, (i) pancreatic $\mathrm{GK}_{\beta}$ is a monomer without allosteric regulation by glucose-6-P; (ii) yeast $\mathrm{Hxk} 2$, like pancreatic $\mathrm{GK}_{\beta}$, has a glucoseregulated subcellular distribution [11, 12], in higher glucose conditions Hxk2 shows a nuclear enrichment that is absent under low-glucose conditions; (iii) both Hxk2 and $\mathrm{GK}_{\beta}$ proteins play a vital role in glucose signalling in the yeast and the pancreatic $\beta$-cell respectively. Current evidence suggests that the main role of S. cerevisiae Hxk2 in the glucose signalling pathway is achieved by its interacting with both Mig1 and Snf1. It has been proposed that Hxk2 inhibits the phosphorylation of the Mig1 repressor, when the cells are growing in high glucose conditions, maintaining in this way the transcriptional repression of target genes $[13,14]$. Since $\mathrm{GK}_{\beta}$ plays a prominent role in pancreatic $\beta$-cell signalling, (inactivation of one glucokinase allele leads to maturityonset diabetes of the young 2 , whereas loss of both alleles is associated with permanent neonatal diabetes $[15,16]), \mathrm{GK}_{\beta}$ is considered as a promising drug target for diabetes therapy. Thus, the study of the regulatory properties of $\mathrm{GK}_{\beta}$ is an important matter to be examined. 
In this work, we took advantage of the facility to manipulate the $S$. cereviside genetic system and the similarity between the yeast Hxk2 and pancreatic $\mathrm{GK}_{\beta}$ to study the role of $\mathrm{GK}_{\beta}$ in the yeast glucose signalling pathway and also to study the regulation of the different subcellular localization that this enzyme presents in yeast cells.

\section{MATERIALS AND METHODS}

\section{Strains and growth media}

GFP and RFP-fluorescence experiments utilized yeast strain H250 (MAT $\alpha$ SUC2 ade2-1 can1-100 his3-11,15 leu2-3,112 trp1-1 ura3-1 mig1- $22:$ LEU2) [17], W303-1A (MATa ura3-52 trp1-289 leu2-3,112 his3- $\Delta 1$ ade2-1 can1-100) [18], DBY1315 (MAT $\alpha$ ura3-52 leu2-3,2-112 lys2-801 gal2) [19], DBY2052 (MAT $\alpha$ hxk1::LEU2 hxk2-202 ura3-52 leu2-3,2-112 lys2-801 gal2) [19], $\Delta$ snf1 [20], $\Delta$ snf1 $\Delta$ hxk1 $\Delta$ hxk2 [20], Y03694 (Mat $\alpha$ his $3 \Delta 1$ leu2 $\Delta 0$ met15 $\Delta 0$ ura3 $\Delta 0$ msn5\%kanMX4) (euroscarf) and FMY388 (MAT $\alpha$ his3 $\Delta 1$ ura3 $\Delta 0$ leu2 $\Delta 0$ met15 $\Delta 0$ MIG1::gfp), the later containing a gfp tagged $M I G 1$ ORF at its chromosomal location. Yeast two-hybrid experiments employed strain

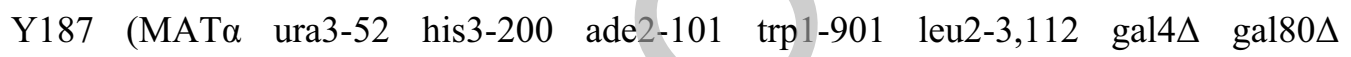
URA3::GAL1 ${ }_{\mathrm{UAS}}-\mathrm{GAL} 1_{\mathrm{TATA}}-\mathrm{lacZ}$ ) [21].

Escherichia coli DH5a (F Ø80dlacZ $\triangle M 15$ recAl endA1 gyrA96 thi-1 hsdR17(r $r_{k^{-}}$ $r_{k^{-}}$) supE44 relA1 deoRA (lacZ YA-argF)U169) was the host bacterial strain for the recombinant plasmid constructions.

Yeast cells were grown in the following media: YEPD, high-glucose ( $2 \%$ glucose, $2 \%$ peptone, and $1 \%$ yeast extract), YEPE, low-glucose $(0.05 \%$ glucose, $3 \%$ ethanol, $2 \%$ peptone, and $1 \%$ yeast extract) and synthetic media containing the appropriate carbon source and lacking appropriate supplements to maintain selection for plasmids ( $2 \%$ glucose $(\mathrm{SD})$; or $3 \%$ ethanol and $0.05 \%$ glucose; and $0.67 \%$ yeast nitrogen base without amino acids). Amino acids and other growth requirements were added at a final concentration of $20-150 \mu \mathrm{g} / \mathrm{ml}$. The solid media contained $2 \%$ agar in addition to the components described above.

\section{Plasimids}

The yeast expression plasmids YEp352-HXK2, YEp352-HXK2/gfp and pWS$\mathrm{GST} / \mathrm{GK}_{\beta}$ were constructed as indicated previously $[13,22]$. 
Plasmids YEp352-GK $\beta$ and pWS93-GK $\beta$ carried a 1398-bp DNA fragment with the complete coding region of human pancreatic glucokinase gene $\left(G K_{\beta}\right)$. Plasmid YEp352-GK $\beta$ was generated by cloning 1398 bp DNA fragment, synthesized by PCR using plasmid pWS-GST/GK $\beta$ [22] as the template and the primer pair OL1 (sense: CAGATAGGATCCATGCTGGACGACAGAGCCAGG) and OL2 (antisense: CTTCAGAATTCCTATCACTGGCCCAGCATACAGGC) into the BamHI-EcoRI site of YEp352-HXK2. The YEp352- GK $\beta$ plasmid expresses the complete coding region of $G K_{\beta}$ gene from the $H X K 2$ promoter. To make plasmid $\mathrm{pWS} 93-\mathrm{GK}_{\beta}$, an EcoRI-SalI fragment obtained from $\mathrm{pWS}-\mathrm{GST} / \mathrm{GK}_{\beta}$ was subcloned into the EcoRI-SalI site of pWS93 vector.

Plasmid YEp352-GK $/$ /gfp and YEp352-GK $\mathrm{GK}_{\mathrm{L}} / \mathrm{gfp}$ were constructed as follows: a 969-bp BamHI-BglII fragment containing the $g f p$ gene was subcloned into YEp352HXK2 plasmid first cleaved with BamHI. Then, a 1398-bp BamHI PCR fragment (OL3: CGTAGGATCCATGGCTATGGATACTACAAG and OL4: GCTAGGATCCCGCCT GGGCCAGCATGCAAGCC) containing the complete coding region of $G K_{\beta}$ gene or a 1398-bp BamHI PCR fragment (OL5: CGTAGGATCCATGGCTATGGA TACTACAAG and OL6: GCTAGGATCCCGCCT GGGCCAGCATGCAAGCC) containing the complete coding region of $G K_{L}$ gene were subcloned into a previously BamHI digested YEp352-HXK2/gfp plasmid. The resulting plasmids express $\mathrm{GK}_{\beta}$ or $\mathrm{GK}_{\mathrm{L}}$ from the $H X K 2$ promoter as fusion proteins with GFP.

For two-hybrid analysis, plasmids pGBKT7-MIG1, pGBKT7-MIG1S311A and pGBKT7-HXK2 were constructed as indicated previously [13, 14, 23]. Plasmids pGBKT7-GK $\beta$ and pGADT7-GK $\beta$ carried a 1398-bp DNA fragment with the complete coding region of $G K_{\beta}$ gene. To make plasmid pGBKT7-GK $\beta$ a EcoRI-SalI fragment obtained from pWS93-GK $\mathrm{K}_{\beta}$ carrying the complete $G K_{\beta}$ gene was subcloned into the EcoRI-SalI sites of pGBKT7. To make plasmid pGADT7-GK $\beta$, an EcoRI-SalI fragment containing the complete coding region of $G K_{\beta}$ gene obtained from $\mathrm{pWS} 93-\mathrm{GK}_{\beta}$ plasmid was subcloned into an EcoRI-XhoI previously cleaved pGADT7 vector. The DNA sequence of all PCR-generated constructs was verified by sequencing and all the clones used were verified by sequencing analysis of fusion points.

\section{Fluorescence microscopy}

Yeast strains expressing the $\mathrm{GK}_{\beta}$-GFP, $\mathrm{GK}_{\mathrm{L}}$-GFP, Hxk2-GFP, Mig1-GFP or 
$\mathrm{Su} 9-\mathrm{RFP}$ fusion proteins were grown to early-log phase $\left(\mathrm{OD}_{600}\right.$ of less than 0.8$)$ in synthetic high-glucose medium (SD-ura). Half of the culture was shifted to synthetic low-glucose medium (SE-ura) for $1 \mathrm{~h}$. The media contained the appropriate carbon source and lacked the appropriate supplements to maintain selection of plasmids. Cells $(25 \mu \mathrm{l})$ were loaded onto poly L-lysine-coated slides, and the remaining suspension was immediately withdrawn by aspiration. One microlitre of DAPI $(2.5 \mu \mathrm{g} / \mathrm{ml}$ in $80 \%$ glycerol) was added, and a covert slide was placed over the microscope slide. GFP, RFP and DAPI localization in live cultures was monitored by direct fluorescence using a Leica DM5000B microscope. To avoid the non-linear range of fluorescent signals, cells highly overexpressing GFP-tagged fusion protein were excluded from further analyses. The localization of proteins was monitored by visual inspection of the images. At least 100 cells were scored in each of at least three independent experiments. The distribution of fluorescence was scored in the following way: $\mathrm{N}$, denotes a nuclear fluorescence signal; C, cytoplasmic fluorescence signal without nuclear or mitochondrial fluorescence signals; $\mathrm{M}$, mitochondrial fluorescence signal.

To stain cells with Mitotracker® Red 580, yeasts were immobilized on poly Llysine-coated slides. Then, they were incubated with $0.5 \mu \mathrm{M}$ MitoTracker ${ }^{\circledR}$ diluted in DMSO and glycerol 80\% (v/v) for $20 \mathrm{~min}$ at room temperature. Finally, MitroTracker ${ }^{\circledR}$ solution was aspired and a glass cover slide was immediately placed over the microscope slide. Images were processed in Adobe Photoshop CS.

\section{Yeast two-hybrid analysis}

The yeast two-hybrid analysis [24] employed yeast vectors pGADT7 and pGBDKT7 and host strain Y187 (described above), in accordance with the Matchmaker two-hybrid system 3 from Clontech. Transformed yeasts were grown in high-glucose (SD/-Leu,Trp) medium. Assays for $\beta$-galactosidase activity followed protocols described elsewhere [25]. Expression levels of the GAD and GBD fusion proteins were controlled by Western blot analysis. Experiments were performed a minimum of three times.

\section{Chromatin immunoprecipitation assay}

Chromatin immunoprecipitation (ChIP) assays were performed essentially as described previously [26]. Cells were harvested and disrupted by vortexing in the presence of glass beads, and the lysate was sonicated to generate DNA fragments that 
ranged in size from 200 to $400 \mathrm{bp}$. To immunoprecipitate HA-tagged proteins, we incubated the extract overnight at $4^{\circ} \mathrm{C}$ with anti-HA antibodies (Santa Cruz Biotechnology, Santa Cruz, CA). To immunoprecipitate Hxk2 protein, we incubated the extract overnight at $4^{\circ} \mathrm{C}$ with anti-Hxk2 antibodies [11]. The sequence primers for PCR to amplify the SUC2 promoter region containing the MIG1 element were, 5'TTATTACTCTGAACAGGA-3' (sense) and 5'-AAGTCGTCAAATCTTTCT-3' (antisense).

\section{RESULTS}

\section{Subcellular localization of human pancreatic $\mathbf{G K}_{\beta}$ in yeast cells}

It has been previously described that human pancreatic glucokinase $\left(\mathrm{GK}_{\beta}\right)$ complements the glucose signalling defect of $S$. cerevisiae $\Delta h x k 2$ mutants, indicating that $\mathrm{GK}_{\beta}$ may substitute Hxk2 in its role of regulating glucose signalling in yeast [22]. To gain insight into the molecular mechanism by which $\mathrm{GK}_{\beta}$ regulates this process in yeast, the glucose-dependent subcellular location of $\mathrm{GK}_{\beta}$ was determined in $S$. cerevisiae cells.

To detect $\mathrm{GK}_{\beta}$ in live yeast cells, a $\mathrm{GK}_{\beta}$-GFP protein fusion was expressed under the control of the $H X K 2$ gene promoter, and its subcellular location was determined by fluorescent microscopy. The $\mathrm{GK}_{\beta}$-GFP fusion protein maintains repression functions in the glucose signalling pathway (data not shown). In cells grown overnight in highglucose, a fraction of $\mathrm{GK}_{\beta}$-GFP was enriched in the nucleus (Fig. 1A and C). Similar results were obtained with an Hxk2-GFP protein fusion, as previously described [13]. However, in cells grown overnight on low-glucose medium, $\mathrm{GK}_{\beta}$-GFP was found mainly associated to multiple organelles distributed through the cytoplasm, reminiscent of a typical mitochondrial network. In contrast, the Hxk2-GFP protein presented an even distribution between the nucleus and the cytoplasm, as previously described [13]

(Fig. 1A). In order to determine whether the $\mathrm{GK}_{\beta}$-GFP protein presented a mitochondrial localization under low-glucose conditions, we labelled the mitochondrial network with a mitotracker probe and observed a similar pattern of subcellular distribution of $\mathrm{GK}_{\beta}$-GFP and the mitotracker (Fig. 1B), suggesting a mitochondrial distribution of $\mathrm{GK}_{\beta}$-GFP under these conditions. To confirm the mitochondrial localization of $\mathrm{GK}_{\beta}$-GFP protein under low-glucose conditions, a red fluorescent protein (RFP) fused with the mitochondrial targeting signal of Neurospora $\mathrm{F}_{0} \mathrm{~F}_{1}$ ATPase subunit 
9 (Su9) [27] was co-expressed with the $\mathrm{GK}_{\beta}$-GFP fusion protein in wild-type cerevisiae cells. As can be seen in Fig. 1B, the expressed proteins were associated with the mitochondria and the fluorescence signals completely overlapped.

Since liver and $\beta$-cell glucokinase isoforms differ only in the first $15 \mathrm{~N}$-terminal residues, it was important to determine if the observed location of $\mathrm{GK}_{\beta}$ was also shared by the liver isoform. As it can be seen in Fig. 1A and C, in cells grown overnight in high-glucose, a fraction of $\mathrm{GK}_{\mathrm{L}}-\mathrm{GFP}$ was enriched in the nucleus and in cells grown overnight on low-glucose medium, $\mathrm{GK}_{\mathrm{L}}$-GFP was found mainly associated to mitochondria (Fig. 1A-C). Therefore, our results suggest that in yeast, the subcellular localization of $\beta$-cell and liver glucokinase isoforms was similar and did not depend on their N-terminus.

\section{The nuclear export of human pancreatic $\mathbf{G K}_{\beta}$ in the yeast cell is Mig1-dependent}

We have recently described that, under low-glucose conditions, Hxk2 exits the nucleus using a pathway in which Mig1 is involved [13, 14]. In order to determine whether Mig1 is also affecting the exit from the nucleus of $\mathrm{GK}_{\beta}$-GFP under the same conditions, we determined the subcellular distribution of $\mathrm{GK}_{\beta}$-GFP in $\Delta m i g 1$ mutant cells (Fig. 2A). The results indicated that $\mathrm{GK}_{\beta}$-GFP fusion protein was enriched in the nucleus both in high and low-glucose-grown cells, suggesting that in the absence of Mig1, GK $\beta_{\beta}$-GFP was not able to exit the nucleus and reach the mitochondria. The introduction in these cells of a plasmid expressing MIG1 under its own promoter (YEp351-MIG1), allowed GK$_{\beta}$-GFP to exit the nucleus under low-glucose conditions (Fig. 2A), as in wild type cells (Fig. 1A), suggesting that in low-glucose conditions, Mig1 is necessary to export the $\mathrm{GK}_{\beta}$-GFP fusion from the nucleus to the mitochondria.

We also studied the subcellular distribution of $\mathrm{GK}_{\beta}$-GFP in a $\Delta h x k 1 \Delta h x k 2$ double mutant strain (Fig. 2B). The results demonstrated that in the absence of Hxk1 and Hxk2, $\mathrm{GK}_{\beta}$-GFP was targeted to the mitochondria both at high and low-glucose concentrations. Since, in the absence of Hxk1 and Hxk2, the protein kinase Snf1 is constitutively activated, we repeated the experiment in a triple $\Delta h x k 1 \Delta h x k 2 \Delta s n f 1$ mutant (Fig 2C). In this case, no mitochondrial distribution of $\mathrm{GK}_{\beta}$-GFP was observed in any growth conditions (similar results were obtained with a single $\Delta s n f l$ mutant, Fig. 2C). These results suggested that $\mathrm{Snf1}$ played a major role in regulating the exit of $\mathrm{GK}_{\beta}$ GFP from the nucleus. When Snf1 was activated, either by growing the cells under low- 
glucose conditions or by deleting negative regulators such as Hxk1 and Hxk2, GK $\beta$ GFP exited the nucleus and presented a mitochondrial distribution.

It is known that Snf1 is involved in the phosphorylation of Mig1 under low-glucose conditions, leading to its exit from the nucleus to the cytoplasm [28]. Since $\mathrm{GK}_{\beta}$-GFP followed the same pattern of distribution as Mig1 and, as we have described above, $\mathrm{GK}_{\beta}$-GFP did not exit the nucleus in the absence of Mig1, even under conditions where Snfl is activated (low-glucose), we suggest that Mig1 could be involved in the exit of $\mathrm{GK}_{\beta}$-GFP from the nucleus under low-glucose conditions. In agreement with this suggestion, we observed that $\mathrm{GK}_{\beta}$-GFP fusion protein was enriched in the nucleus, in both high and low-glucose-grown cells, in the absence of Msn5 (Fig. 3A), a member of the importin family of nuclear transport proteins which is required to export Mig1 from the nucleus in low glucose conditions [29]. This result suggests that in the absence of Msn5, GK $\beta_{\beta}$-GFP was not able to exit the nucleus and reach the mitochondria. Thus, taken together, these data demonstrated that Mig1 and $\mathrm{GK}_{\beta}$-GFP could form part of a nuclear complex whose export to the cytoplasm was dependent on both, the phosphorylation of Mig1 by Snf1 and the exportin Msn5. The close relationship between Mig1 and $\mathrm{GK}_{\beta}$-GFP was confirmed when we observed that a Mig1-GFP protein fusion was dragged to the mitochondrial network, under low-glucose conditions, only if $\mathrm{GK}_{\beta}$ was expressed in the same cells (Fig. 3B). These results suggested that $\mathrm{Mig} 1$ and $\mathrm{GK}_{\beta}$ may form a complex in which Mig1 confers the determinants for nuclear export and $\mathrm{GK}_{\beta}$ confers the determinants for mitochondrial localization under lowglucose conditions.

\section{Human pancreatic $\mathrm{GK}_{\beta}$ interacts with the yeast Mig1 transcriptional repressor}

The experiments described above strongly suggest that Mig1 and $\mathrm{GK}_{\beta}$ may form part of an in vivo complex. To confirm the possible physical interaction between Mig1

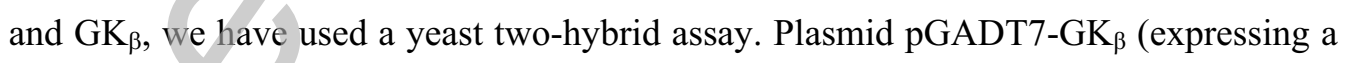
fusion of the Gal4 activating domain, $\mathrm{GAD}$, to $\mathrm{GK}_{\beta}$ ) was co-transformed with a plasmid expressing a fusion of the Gal4 binding domain (GBD) with either Hxk2, Mig1, Mig1S311A or $\mathrm{GK}_{\beta}$, into an appropriate reporter strain. The interaction between the selected proteins was monitored by measurement of the $\beta$-galactosidase activity. As shown in Fig. 4, the GAD-GK ${ }_{\beta}$ fusion protein produced a strong interaction with GBD- 
Mig1. However, this interaction was absent in a GBD-Mig1S311A mutant, indicating that the Ser311 residue of Mig1, previously identified as essential for Hxk2 interaction [30], was also required for interaction with $\mathrm{GK}_{\beta}$. No interaction was observed between $\mathrm{GAD}_{-\mathrm{GK}_{\beta}}$ and GBD-Hxk2 or GBD-GK$\beta$, indicating that these proteins did not form dimers. Similar levels of all protein fusions were detected in all the cases (not shown). To confirm the interaction we performed co-immunoprecipitation and GST pull-down assays but we were not able to observe any interaction between $\mathrm{GK}_{\beta}$ and Mig1, probably because it was too weak or transient to be detected by these methods.

\section{Human pancreatic $\mathrm{GK}_{\beta}$ binds in vivo to the $S U C 2-\mathrm{Mig} 1$ repressor complex}

Previous reports have demonstrated that Hxk2 participates in the SUC2-Mig1 repressor complex by interaction with Mig1 protein and not by direct binding to DNA $[14,31,32]$. As $\mathrm{GK}_{\beta}$ and Mig1 may form an in vivo complex (see above), we investigated the biological significance of this interaction by determining whether $\mathrm{GK}_{\beta}$ localized in the SUC2-Mig1 repressor complex in a glucose dependent manner. To test this hypothesis, we used ChIP assays. Our results show that in cells grown in high glucose medium, both $\mathrm{HA}-\mathrm{GK}_{\beta}$ and $\mathrm{Hxk} 2$ proteins were recruited to a DNA fragment of the SUC2 promoter containing the Mig1 binding site (Fig. 5A, lanes 1 and 3). Conversely, in low glucose medium, HA-GK ${ }_{\beta}$ and Hxk2 binding to the SUC2-Mig1 complex was abolished (Fig. 5B, lanes 2 and 4). Binding of $\mathrm{HA}-\mathrm{GK}_{\beta}$ to the Mig1 site of the SUC2 promoter was dependent on the presence of Mig1, since in $\triangle m i g 1$ cells, no binding of $\mathrm{HA}-\mathrm{GK}_{\beta}$ to the $S U C 2$ promoter was observed under any growth condition (Fig. 5B). No DNA amplification was observed when we used cells with untagged $\mathrm{GK}_{\beta}$ or cells with HA-tagged Rgtl protein (data not shown).

Taking all these results together we suggest that $\mathrm{GK}_{\beta}$ forms a similar complex with Mig1 as Hxk2 does, and that this complex is involved in the glucose regulated expression of the SUC2 gene. This complex exits the nucleus under conditions of Snf1 activation, being the presence of Mig1 and Msn5 necessary for the nuclear exit.

\section{DISCUSSION}

In this study, we have taken advantage of the facility to manipulate the $S$. cereyisiae genetic system to determine the mechanisms by which $\mathrm{GK}_{\beta}$ regulates glucose 
signalling in yeast cells and to examine the glucose-dependent localization of the $\mathrm{GK}_{\beta}$ in yeasts.

Reports suggest that $\mathrm{Hxk} 2$, the yeast orthologue of $\mathrm{GK}_{\beta}$, has a dual function in yeast cells, first initiating the intracellular metabolism of glucose by its enzymatic activity and secondly, signalling glucose repression by its interaction with nuclear Mig1 repressor, regulating its phosphorylation status [14]. Since Hxk2 protein mutations without phosphorylating activity but retaining activity in glucose repression signalling have not been achieved [33,34], the idea that the sugar kinase activity and the sugar signalling properties are mediated through separate domains of Hxk2 is still controversial. In this study, we demonstrate that recombinant human pancreatic $\mathrm{GK}_{\beta}$ is able to signal glucose repression in S. cerevisiae; both $\mathrm{GK}_{\beta}$ and $\mathrm{Hxk} 2$ have a nuclear location during growth in high-glucose conditions and both are able to regulate the glucose-responsive $S U C 2$ gene in $S$. cerevisiae. We also report that $\mathrm{GK}_{\beta}$ is physically associated with the Mig1 protein repressor and ChIP assays confirmed that both $\mathrm{GK}_{\beta}$ and Hxk2 interacted with Mig1 in a cluster with DNA fragments containing the MIG1 site of the SUC2 promoter. Therefore, our results suggest that $\mathrm{GK}_{\beta}$ and Hxk2 use similar mechanisms to regulate glucose signalling in yeast.

The other important finding of this study regards the subcellular location of $\mathrm{GK}_{\beta}$. We report that $\mathrm{GK}_{\beta}$ moves between the nucleus and mitochondria in the yeast cell in response to glucose concentration in the medium: under high-glucose conditions, $\mathrm{GK}_{\beta}$ is enriched in the nucleus and resides there in association with Mig1; however, under low-glucose conditions, $\mathrm{GK}_{\beta}$ exits the nucleus and eventually reaches the mitochondria, either because it has uncharacterized mitochondrial determinants $\left(\mathrm{GK}_{\beta}\right.$, lacks the hydrophobic N-terminal sequence present in hexokinases I and II that allows their binding to the mitochondria [35]), or because it interacts with another protein that has these mitochondrial localization determinants, as in the case of mammalian proapoptotic factor $\mathrm{BAD}$ which is involved in the mitochondrial targeting of glucokinase in both liver and pancreatic beta-cells [6]. To reach $\mathrm{GK}_{\beta}$ this subcellular location, first, Mig1 must be present in the cell and Snf1 must be active. Then, binding of Mig1 to $\mathrm{GK}_{\beta}$ allows the Mig1-GK $\mathrm{GK}_{\beta}$ complex to exit the nucleus by a mechanism dependent on the exportin Msn5. Finally, the complex is located in the mitochondrial network, location that has never been reported for either Mig1 or Hxk2. 
Since the subcellular distribution of glucokinase in mammalian pancreatic $\beta$-cell is also regulated by glucose: low levels of glucose causes an association of GK with mitochondria [8], whereas, high glucose levels prevents GK translocation to this organelle [8], the yeast system offers a possibility to study the nature of the determinants present in $\mathrm{GK}_{\beta}$ or the identification of interactive proteins that target $\mathrm{GK}_{\beta}$ to the mitochondria under low-glucose conditions.

The role of Mig1 in regulating $\mathrm{GK}_{\beta}$ exit from the nucleus could be compared to the role of the glucokinase regulatory protein (GKRP) in mammalian cells $[4,5]$. However these two proteins present different domain structures and only 17\% similarity (assessed by CLUSTAL analysis), suggesting that the relationship between the two proteins is only at the level of their interaction with GK

Altogether, our results indicate that despite the strong phylogenetic difference between the human $\mathrm{GK}_{\beta}$ and the yeast Hxk2 proteins, the mechanism of glucose signalling is maintained, suggesting that the function of the regulatory domain of these proteins has been conserved throughout evolution.

\section{ACKNOWLEDGEMENTS}

We are very grateful to J. Guinoyart for fruitful discussions. This work was supported by grants BFU2004-02855-C02-02 and BFU2007-66063-C02-02/BMC from the MEC-DGI (Spain). A.R. was supported by MEC-DGI (Spain). D.A. was supported by the Fundación Carolina (Spain). This work was also supported by grants from the CIBER de Enfermedades Raras, an initiative of the ISCIII, to P.S. 


\section{REFERENCES PUNTEAR LAS ABREVIATURAS DE LAS REVISTAS}

1 Wilson, J. E. (1995) Hexokinases. Rev. Physiol. Biochem. Pharmacol., 126, 65198

2 Agius, L., Peak, M., Newgard, C. B., Gomez-Foix, A. M. and Guinovart, J. J.

(1996) Evidence for a role of glucose-induced translocation of glucokinase in the control of hepatic glycogen synthesis. J. Biol. Chem., 271, 30479-30486

3 Matschinsky, F. M. (2005) Glucokinase, glucose homeostasis, and diabetes mellitus. Curr. Diab. Rep., 5, 171-176

4 de la Iglesia, N., Veiga-da-Cunha, M., Van Schaftingen, E., Guinovart, J. J. and Ferrer, J. C. (1999) Glucokinase regulatory protein is essential for the proper subcellular localisation of liver glucokinase. FEBS Lett., 456, 332-338

5 Veiga-da-Cunha, M. and Van Schaftingen, E. (2002) Identification of fructose 6phosphate- and fructose 1-phosphate-binding residues in the regulatory protein of glucokinase. J. Biol. Chem., 277, 8466-8473

6 Danial, N. N., Gramm, C. F., Scorrano, L., Zhang, C. Y., Krauss, S., Ranger, A. M., Datta, S. R., Greenberg, M. E., Licklider, L. J., Lowell, B. B., Gygi, S. P. and Korsmeyer, S. J. (2003) BAD and glucokinase reside in a mitochondrial complex that integrates glycolysis and apoptosis. Nature, 424, 952-956

7 Arden, C., Baltrusch, S. and Agius, L. (2006) Glucokinase regulatory protein is associated with mitochondria in hepatocytes. FEBS Lett., 580, 2065-2070

8 Kim, W. H., Lee, J. W., Suh, Y. H., Hong, S. H., Choi, J. S., Lim, J. H., Song, J. H., Gao, B. and Jung, M. H. (2005) Exposure to chronic high glucose induces beta-cell apoptosis through decreased interaction of glucokinase with mitochondria: downregulation of glucokinase in pancreatic beta-cells. Diabetes, 54, 2602-2611

9 Rizzo, M. A. and Piston, D. W. (2003) Regulation of beta cell glucokinase by Snitrosylation and association with nitric oxide synthase. J. Cell. Biol., 161, 243-248

10 Arden, C., Harbottle, A., Baltrusch, S., Tiedge, M. and Agius, L. (2004) Glucokinase is an integral component of the insulin granules in glucose-responsive insulin secretory cells and does not translocate during glucose stimulation. Diabetes, $\mathbf{5 3}$, 2346-2352

11 Randez-Gil, F., Herrero, P., Sanz, P., Prieto, J. A. and Moreno, F. (1998) Hexokinase PII has a double cytosolic-nuclear localisation in Saccharomyces cerevisiae. FEBS Lett., 425, 475-478

12 Stubbs, M., Aiston, S and Agius, L. (2000) Subcellular localization, mobility, and kinetic activity of glucokinase in glucose-responsive insulin-secreting cells. Diabetes, 49, 2048-2055

13 Ahuatzi, D., Herrero, P., de la Cera, T. and Moreno, F. (2004) The glucoseregulated nuclear localization of hexokinase 2 in Saccharomyces cerevisiae is Mig1dependent. J. Biol. Chem., 279, 14440-14446

14 Ahuatzi, D., Riera, A., Pelaez, R., Herrero, P. and Moreno, F. (2007) Hxk2 regulates the phosphorylation state of Mig1 and therefore its nucleocytoplasmic distribution. J. Biol. Chem., 282, 4485-4493

15 Vionnet, N., Stoffel, M., Takeda, J., Yasuda, K., Bell, G. I., Zouali, H., Lesage, S., Velho, G., Iris, F., Passa, P. and et al. (1992) Nonsense mutation in the glucokinase gene causes early-onset non-insulin-dependent diabetes mellitus. Nature, 356, 721-722 16 Njolstad, P. R., Sovik, O., Cuesta-Munoz, A., Bjorkhaug, L., Massa, O., Barbetti, F., Undlien, D. E., Shiota, C., Magnuson, M. A., Molven, A., Matschinsky, F. M. and Bell, G. I. (2001) Neonatal diabetes mellitus due to complete glucokinase deficiency. N. Engl. J. Med., 344, 1588-1592 
17 Nehlin, J. O. and Ronne, H. (1990) Yeast MIG1 repressor is related to the mammalian early growth response and Wilms' tumour finger proteins. EMBO J., 9, 2891-2898

18 Wallis, J. W., Chrebet, G., Brodsky, G., Rolfe, M. and Rothstein, R. (1989) A hyper-recombination mutation in S. cerevisiae identifies a novel eukaryotic topoisomerase. Cell, $\mathbf{5 8}, 409-419$

19 Ma, H. and Botstein, D. (1986) Effects of null mutations in the hexokinase genes of Saccharomyces cerevisiae on catabolite repression. Mol. Cell. Biol., 6, 4046-4052

20 Tomas-Cobos, L. and Sanz, P. (2002) Active Snf1 protein kinase inhibits expression of the Saccharomyces cerevisiae HXT1 glucose transporter gene. Biochem. J., 368, 657-663

21 Harper, J. W., Adami, G. R., Wei, N., Keyomarsi, K. and Elledge, S. J. (1993) The p21 Cdk-interacting protein Cip1 is a potent inhibitor of G1 cyclin-dependent kinases. Cell, 75, 805-816

22 Mayordomo, I. and Sanz, P. (2001) Human pancreatic glucokinase (GlkB) complements the glucose signalling defect of Saccharomyces cerevisiae hxk2 mutants. Yeast, 18, 1309-1316

23 de la Cera, T., Herrero, P., Moreno-Herrero, F., Chaves, R. S. and Moreno, F. (2002) Mediator factor Med8p interacts with the hexokinase 2: implication in the glucose signalling pathway of Saccharomyces cerevisiae. J. Mol. Biol., 319, 703-714

24 Fields, S. and Song, O. (1989) A novel genetic system to detect protein-protein interactions. Nature, 340, 245-246

25 Rupp, S. (2002) LacZ assays in yeast. Methods Enzymol., 350, 112-131

26 Palomino, A., Herrero, P. and Moreno, F. (2006) Tpk3 and Snf1 protein kinases regulate Rgt1 association with Saccharomyces cerevisiae HXK2 promoter. Nucleic Acids Res., 34, 1427-1438

27 Westermann, B. and Neupert, W. (2000) Mitochondria-targeted green fluorescent proteins: convenient tools for the study of organelle biogenesis in Saccharomyces cerevisiae. Yeast, 16, 1421-1427

28 De Vit, M. J., Waddle, J. A. and Johnston, M. (1997) Regulated nuclear translocation of the Mig1 glucose repressor. Mol. Biol. Cell., 8, 1603-1618

29 DeVit, M. J. and Johnston, M. (1999) The nuclear exportin Msn5 is required for nuclear export of the Mig1 glucose repressor of Saccharomyces cerevisiae. Curr. Biol., 9, 1231-1241

30 Ahuatzi, D., Riera, A., Pelaez, R., Herrero, P. and Moreno, F. (2006) Hxk2 regulates the phosphorylation state of Mig1 and therefore its nucleocytoplasmic distribution. J. Biol. Chem., 282, 4485-4493

31 Herrero, P., Martinez-Campa, C. and Moreno, F. (1998) The hexokinase 2 protein participates in regulatory DNA-protein complexes necessary for glucose repression of the SUC2 gene in Saccharomyces cerevisiae. FEBS Lett., 434, 71-76

32 Moreno, F., Ahuatzi, D., Riera, A., Palomino, C. A. and Herrero, P. (2005) Glucose sensing through the Hxk2-dependent signalling pathway. Biochem. Soc. Trans., 33, 265-268

33 Hohmann, S., Winderickx, J., de Winde, J. H., Valckx, D., Cobbaert, P., Luyten, K., de Meirsman, C., Ramos, J. and Thevelein, J. M. (1999) Novel alleles of yeast hexokinase PII with distinct effects on catalytic activity and catabolite repression of SUC2. Microbiology, 145 ( Pt 3), 703-714

34 Mayordomo, I. and Sanz, P. (2001) Hexokinase PII: structural analysis and glucose signalling in the yeast Saccharomyces cerevisiae. Yeast, 18, 923-930 
35 Wilson, J. E. (2003) Isozymes of mammalian hexokinase: structure, subcellular localization and metabolic function. J. Exp. Biol., 206, 2049-2057 


\section{FIGURE LEGENDS}

Figure 1. Glucose regulates the subcellular localization of both Hxk2-GFP and GK $\mathbf{\beta}^{-G F P}$

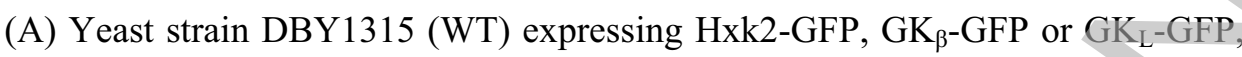
from plasmids YEp352-HXK2/gfp, YEp352-GK $\beta$ /gfp or YEp352-GK $/$ gfp respectively, was grown on high-glucose synthetic medium (H-Glc) until an OD $600 \mathrm{~nm}$ of 1.0 was reached and then transferred to low glucose synthetic medium (L-Glc) for $60 \mathrm{~min}$. Then, cells were stained with DAPI and imaged for GFP and DAPI fluorescence. (B) Cells growing under low-glucose conditions and expressing $\mathrm{GK}_{\beta}-\mathrm{GFP}$ were stained with Mitotracker ${ }^{\circledR}$ Red 580. Cells growing under low-glucose conditions and expressing both $\mathrm{GK}_{\beta}$-GFP and Su9-RFP were stained with DAPI and imaged for DAPI, GFP and RFP fluorescence. (C) The localization of fluorescent reporter proteins was determined in at least 100 cells in three independent experiments; $\mathrm{N}$, denotes a nuclear fluorescence signal; C, cytoplasmic fluorescence signal without nuclear or mitochondrial fluorescence signals; M, mitochondrial fluorescence signal. Means and standard deviations are shown for at least three independent experiments.

\section{Figure 2. Mig1 and Snf1 regulate the subcellular localization of $G_{\beta}-G F P$}

(A) Yeast strain $\mathrm{H} 250$ (Amig1), expressing $\mathrm{GK}_{\beta}$-GFP from a plasmid (YEp352$\mathrm{GK}_{\beta} / \mathrm{gfp}$ ) was transformed or not with plasmid YEp351-MIG1. Transformants were grown on high-glucose synthetic medium (H-Glc) until an OD 600nm of 1.0 and then transferred to medium with low-glucose synthetic medium (L-Glc) for $60 \mathrm{~min}$. Then, cells were stained with DAPI and imaged for GFP and DAPI fluorescence. (B) Yeast strains DBY1315 (WT) and DBY2052 ( $\Delta h x k 1 \Delta h x k 2)$ and (C) yeast strains $\Delta s n f 1$ and $\Delta s n f 1 \Delta h x k 1 \Delta h x k 2$, expressing $\mathrm{GK}_{\beta}$-GFP from a plasmid YEp352- $\mathrm{GK}_{\beta} / \mathrm{gfp}$, were analyzed in the same way.

Figure 3. Msn5 directs the nuclear export of $\mathbf{G K}_{\beta}$ and $\mathbf{G K}_{\beta}$ directs mitochondrial localization of Mig1.

(A) Yeast strain Y03694 ( $\Delta m s n 5)$, expressing Mig1-GFP or $\mathrm{GK}_{\beta}$-GFP from plasmids YEp352-MIG1/gfp or YEp352- $\mathrm{GK}_{\beta} /$ gfp was grown on high-glucose synthetic medium (H-Glc) until an OD $600 \mathrm{~nm}$ of 1.0 and then transferred to medium with lowglucose synthetic medium (L-Glc) for $60 \mathrm{~min}$. Then, cells were stained with DAPI and 
imaged for GFP and DAPI fluorescence. (B) Yeast strain FMY388 expressing a $g f p$ tagged MIGl ORF at its chromosomal location, was transformed or not with plasmid YEp352-GK ${ }_{\beta}$. Transformants were analysed as indicated in part A.

\section{Figure 4. Two-hybrid interaction between $\mathrm{GK}_{\beta}$ and Mig1}

Plasmids containing full-length Hxk2, Mig1, Mig1S311A and $\mathrm{GK}_{\beta}$ fused to the Gal4 DNA binding domain (GBD) and the empty plasmid were co-transformed into yeast strain Y187 with a construct encoding the Gal4 activating domain (GAD) fused to full-length $\mathrm{GK}_{\beta}$. Transformants were grown on high-glucose synthetic medium (H-Glc) and protein-protein interactions were examined by measuring the $\beta$-galactosidase activity. Values are averages of $\beta$-galactosidase activities for three transformants, with a standard deviation of less than $10 \%$ in all the cases.

\section{Figure 5. In vivo binding of $\mathrm{GK}_{\beta}$ to the MIG1 element of the SUC2 promoter}

Wild type W303-1A (A) and $\Delta m i g 1$ mutant cells (B) containing a HA-tagged version of $\mathrm{GK}_{\beta}$ (from plasmid pWS-GST/GK ) were grown in high (H-Glc) or low (LGlc) glucose conditions. Cell extracts were prepared and immunoprecipitated with antiHA or anti-Hxk2 antibodies and the DNA fragments were amplified by PCR using the combination of oligonucleotides indicated in Materials and Methods. The amplified fragments were resolved by agarose gel electrophoresis. Migration of standard markers is indicated on the left. A representative ChIP assay out of three independent experiments is shown. 


\section{B Biochemical Journal Immediate Publication. Published on 30 Jun 2008 as manuscript BJ20080797}

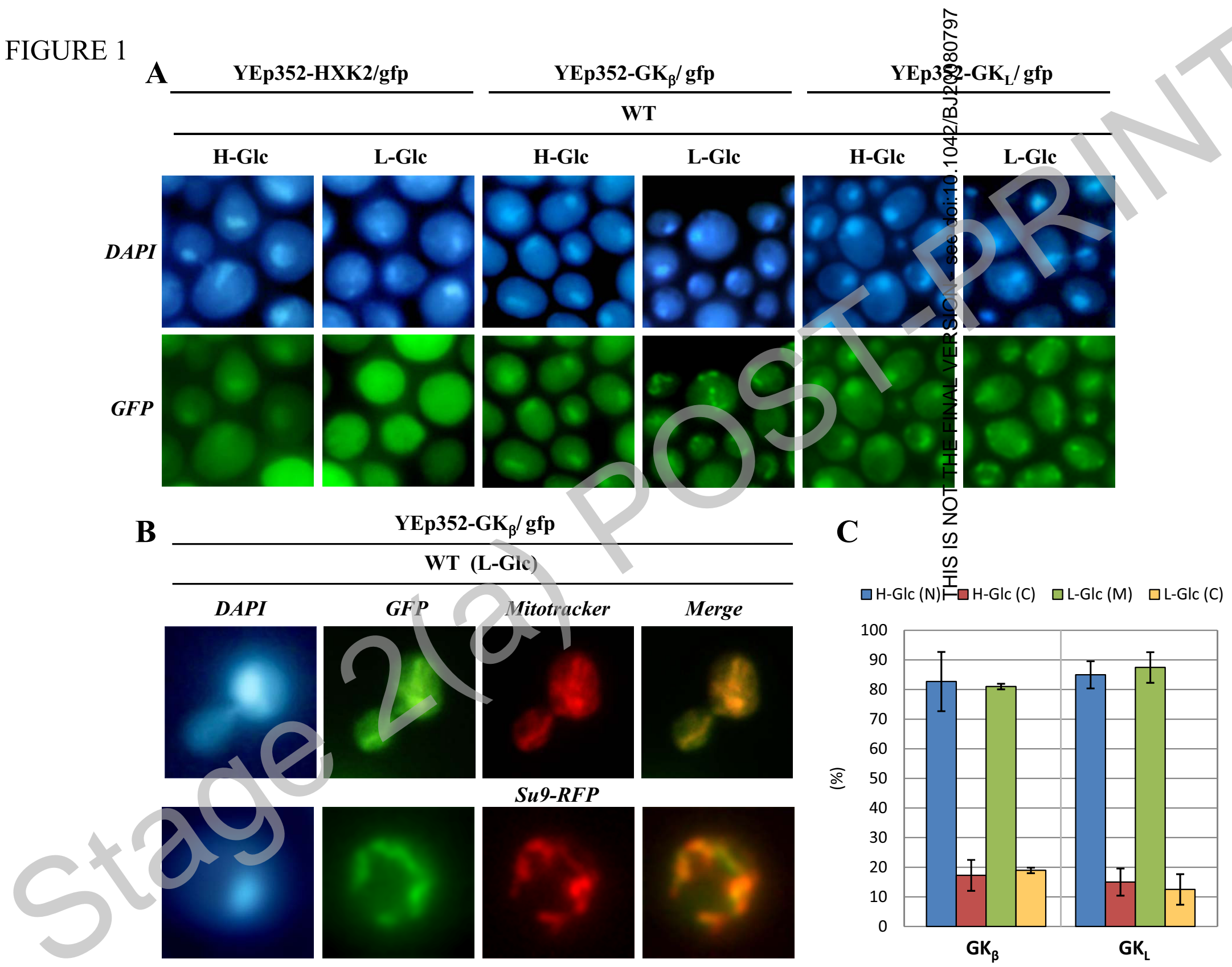

Licenced copy. Copying is not permitted, except with prior permission and as allowed by law. (c) 2008 The Authors Journal compilation (c) 2008 Biochemical Society 


\section{FIGURE 2}

A

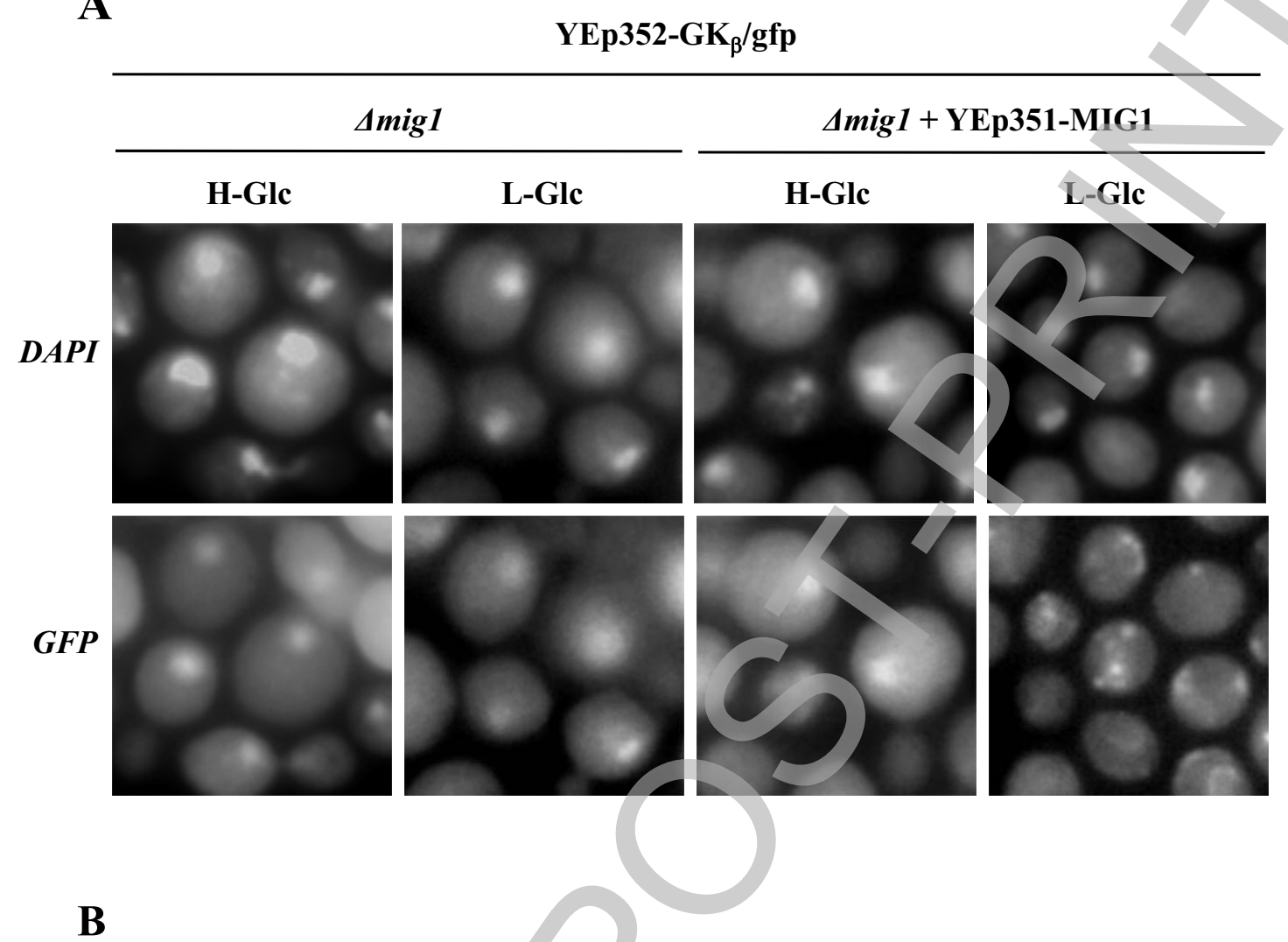

YEp352-GK $/$ gfp

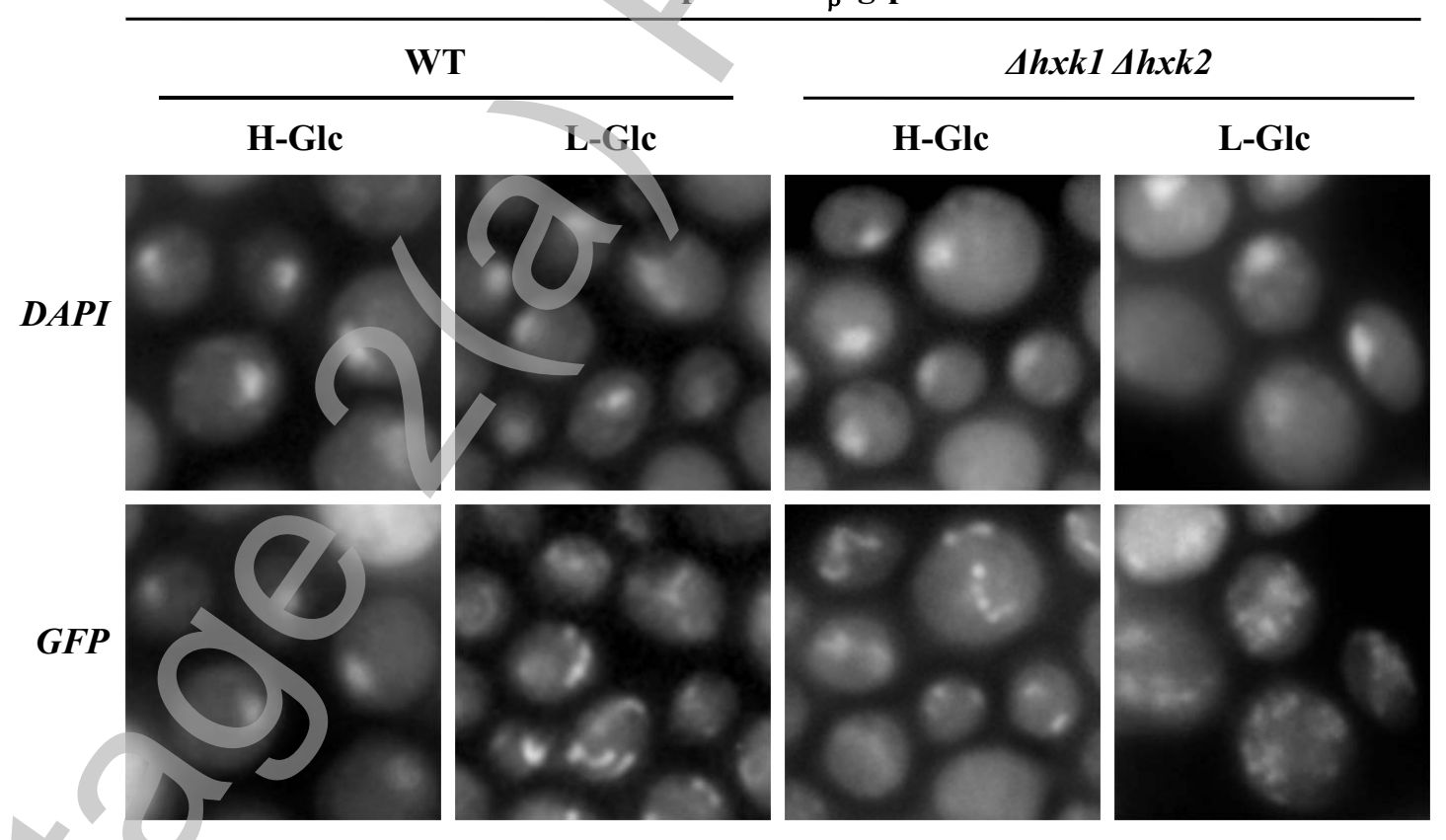

Licenced copy. Copying is not permitted, except with prior permission and as allowed by law. (C) 2008 The Authors Journal compilation (C) 2008 Biochemical Society 


\section{FIGURE 2}

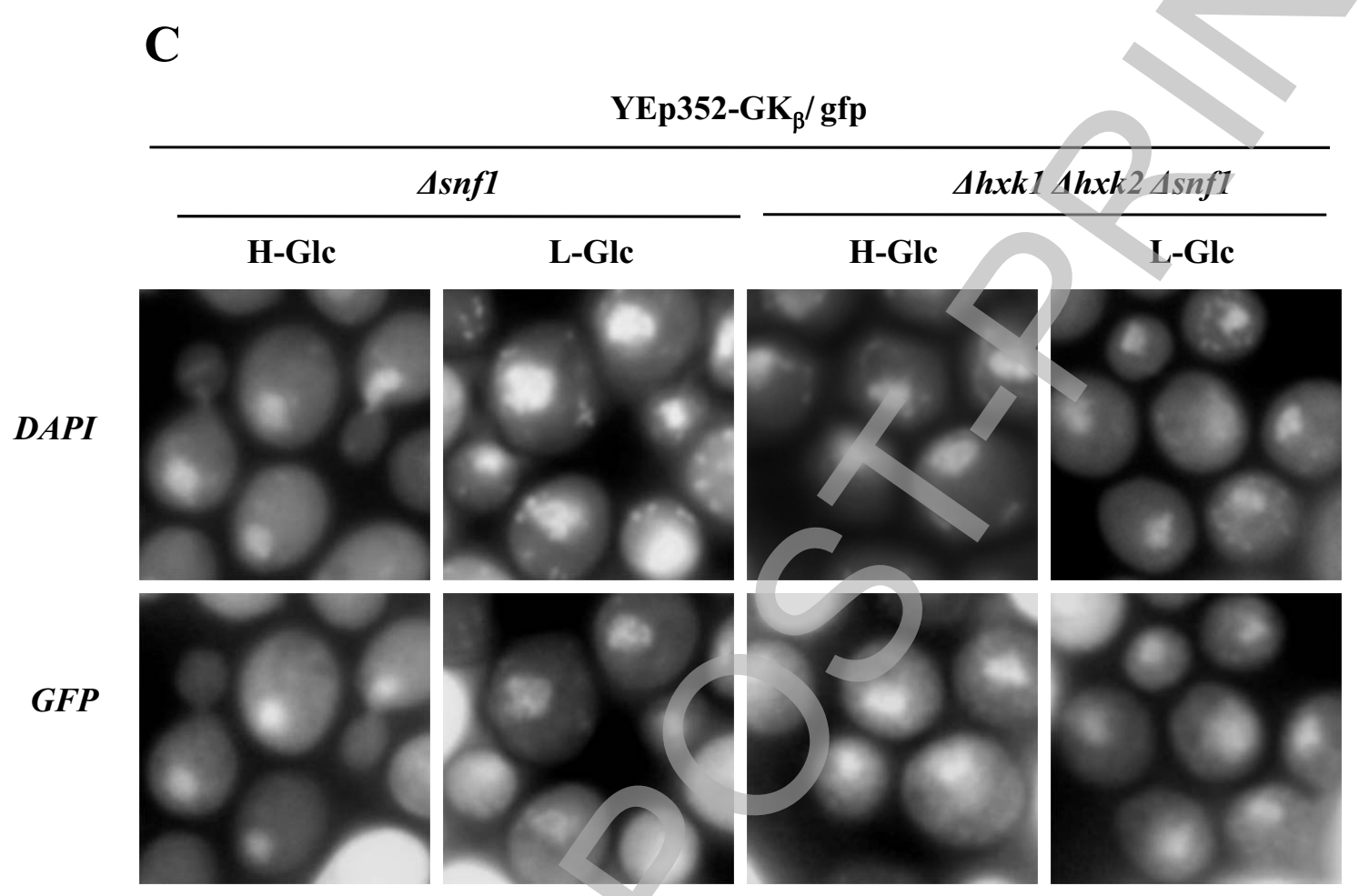

Licenced copy. Copying is not permitted, except with prior permission and as allowed by law. (C) 2008 The Authors Journal compilation (C) 2008 Biochemical Society 
B Biochemical Journal Immediate Publication. Published on 30 Jun 2008 as manuscript BJ20080797

\section{FIGURE 3}
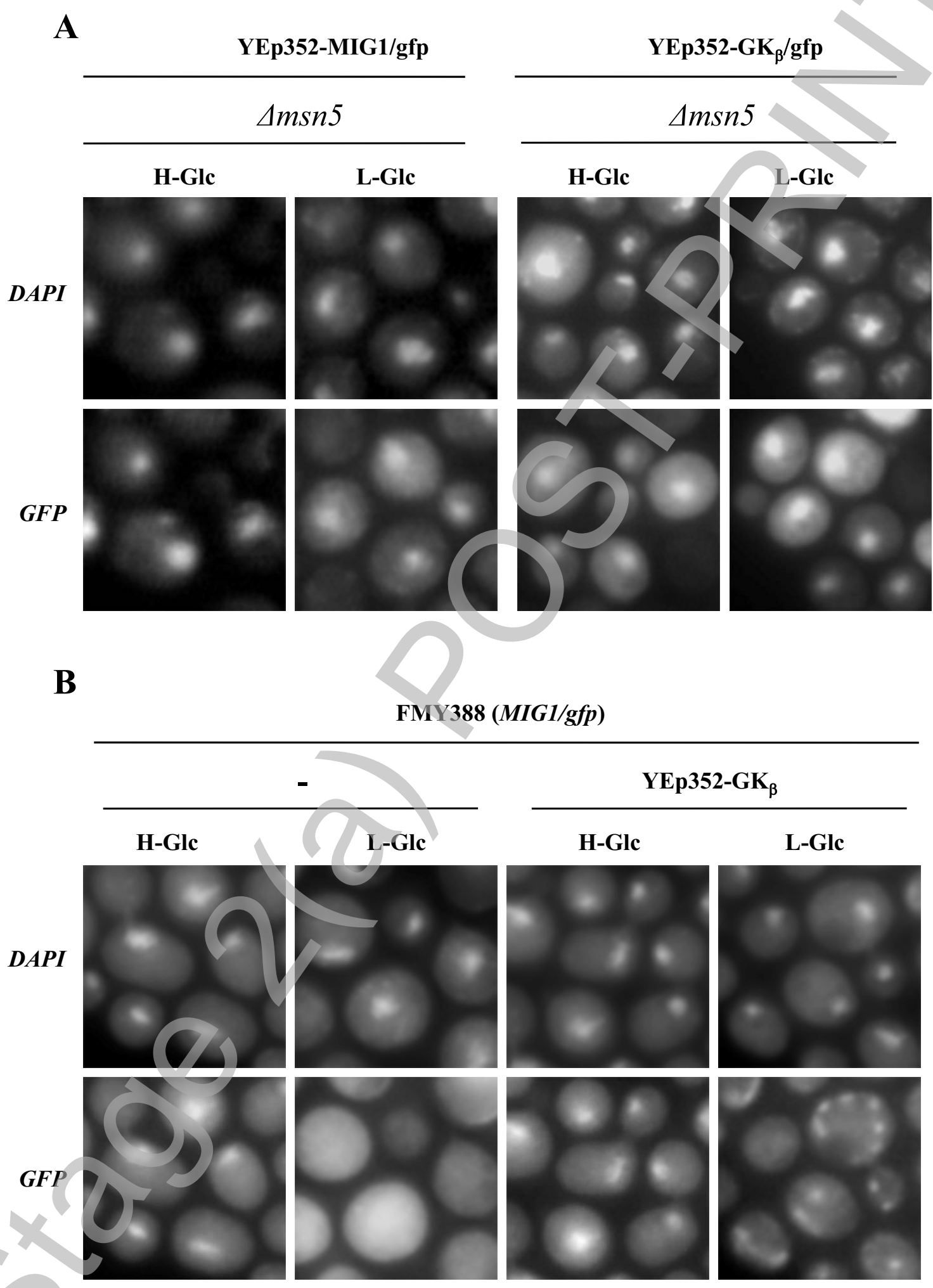

Licenced copy. Copying is not permitted, except with prior permission and as allowed by law. (C) 2008 The Authors Journal compilation (C) 2008 Biochemical Society 


\section{FIGURE 4}

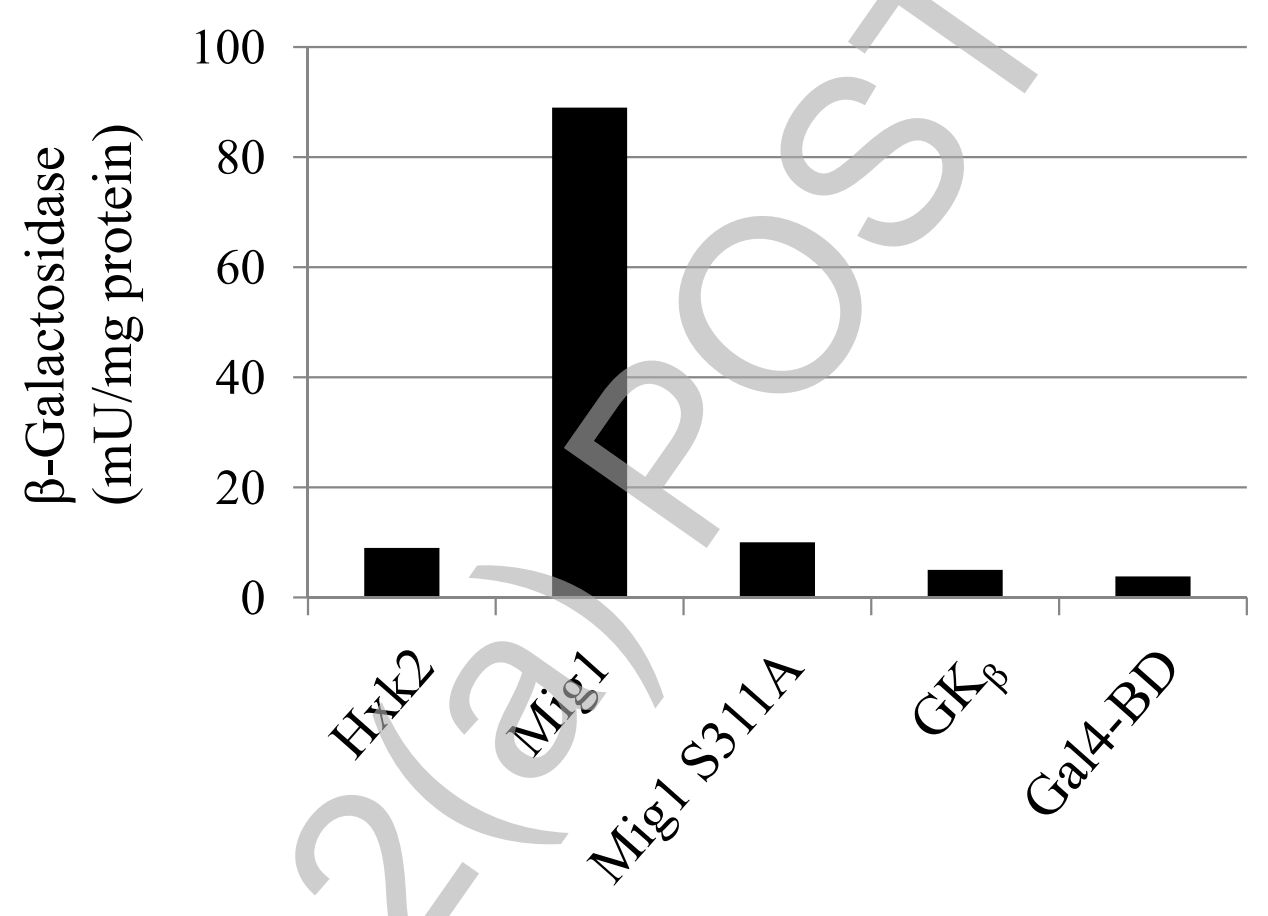




\section{FIGURE 5}

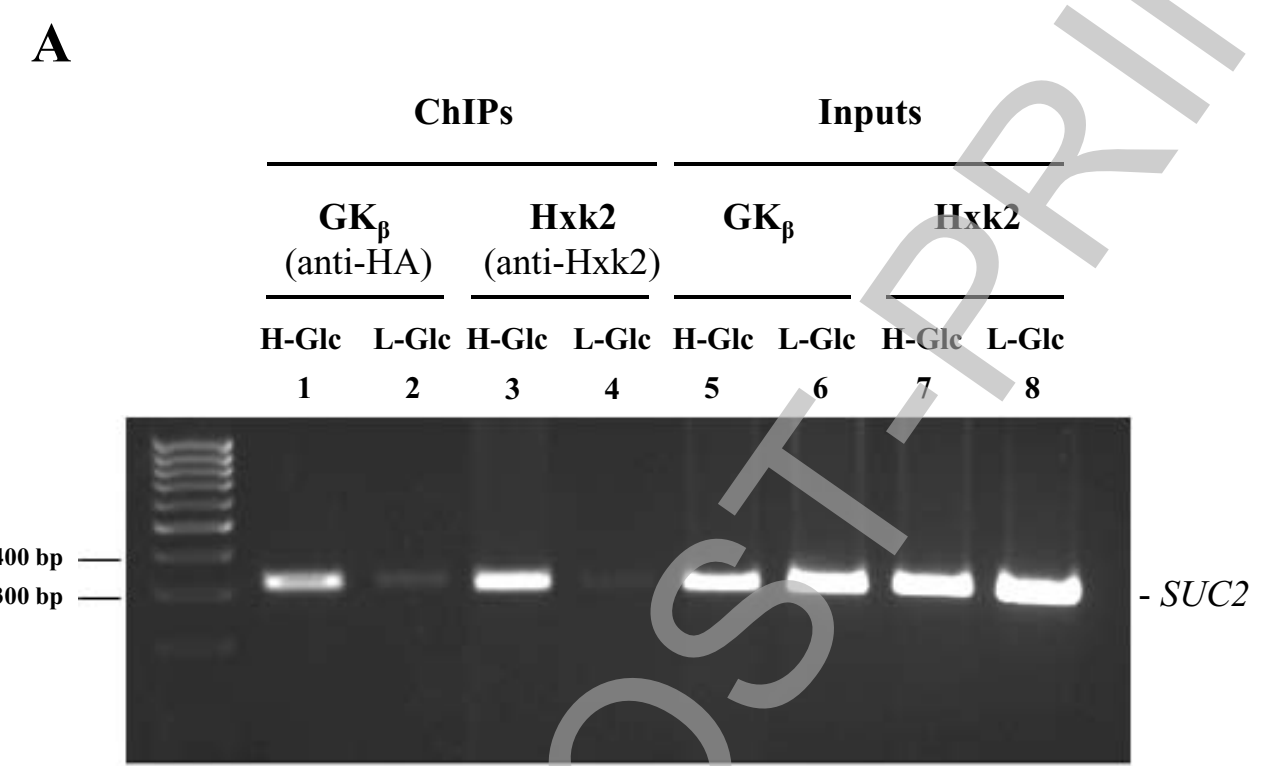

B

\section{ChIPs}

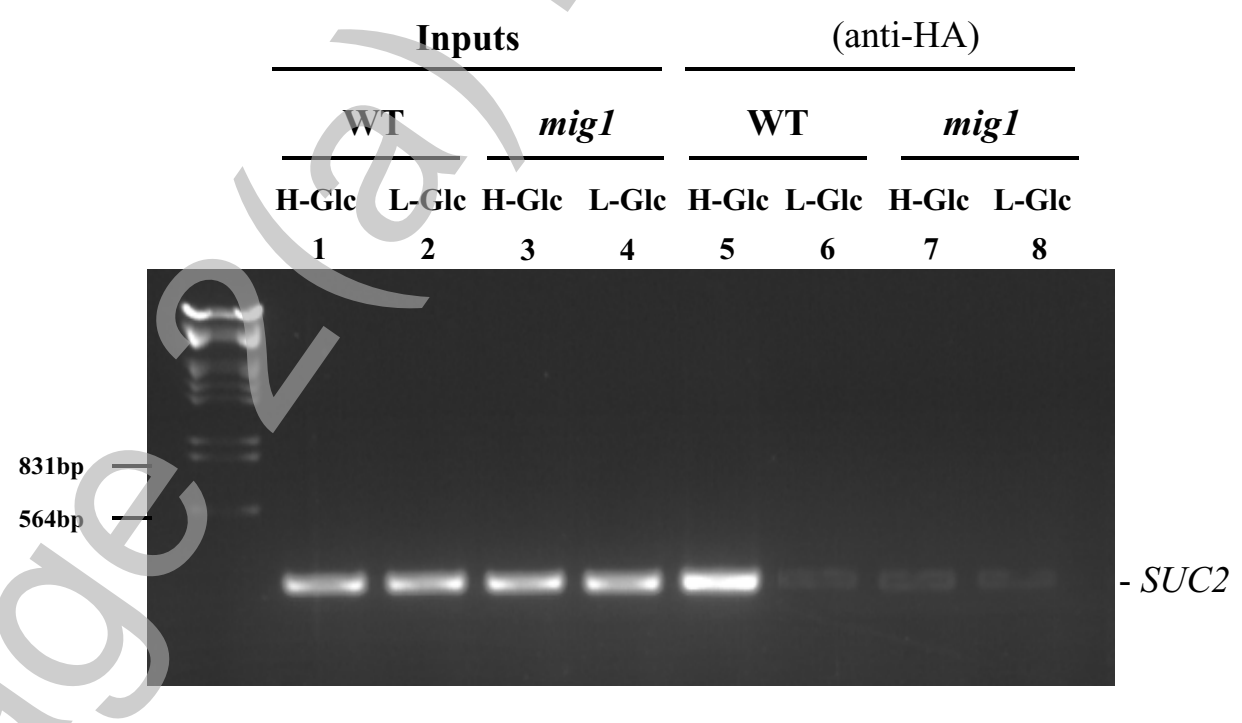

